\title{
Metamaterial Tunable Filter Design
}

\author{
Naima Benmostefa ${ }^{1}$, M. Meliani ${ }^{1}$, H. Ouslimani ${ }^{2}$ \\ ${ }^{1}$ STIC Laboratory, Department of Electrical Engineering, Faculty of Technology, University Abou Bekr Belkaïd-Tlemcen, Tlemcen, \\ Algeria; ${ }^{2}$ University Paris Ouest Nanterre la Défense, Paris, France. \\ Email: benmostefanaima@yahoo.fr
}

Received February $4^{\text {th }}, 2013$; revised March $8^{\text {th }}, 2013$; accepted March $15^{\text {th }}, 2013$

Copyright (C) 2013 N. Benmostefa et al. This is an open access article distributed under the Creative Commons Attribution License, which permits unrestricted use, distribution, and reproduction in any medium, provided the original work is properly cited.

\begin{abstract}
This paper presents a new concept to implement a tunable filter metamaterial with dual negative refraction composed of ferrite slabs and metallic resonators, including split-ring resonators (SRR), and short wire pairs. The ferrite slabs under an applied magnetics bias provide one magnetic resonance frequency band and the metallic resonators provide another one. The continuous wires within the metamaterials provide the negative permittivity in a wide frequency band covering the two magnetic resonance bands. This type of metamaterialis analyzed in detail, and tunable stop band filters are successfully designed. The effective electromagnetic parameters obtained from the simulation of the S-parameters indicate that metamaterial exhibit negative refraction bands, which can be shifted by changing the magnetic bias.
\end{abstract}

Keywords: Metamaterial; SRR; CSRR; Tunable; Filter; Magnetic Bias

\section{Introduction}

Metamaterial is one type of material who possesses abnormal property that not found in nature which has opened a promising realm for physics as well as for engineering [1]. From the original work of negative refraction in Microwave frequency based on simultaneously negative effective permittivity and permeability to recent research on electromagnetic (EM), metamaterial has generated great interests with the potential to manipulate EM waves [2,3].

However, the property of metamaterial makes it generally an inactive device, which seems to be a bottleneck for practical applications.

However, considerable researches have previously been devoted to the tunability of the metamaterial LHM (Left Hand media). It is known that an ideal single Split Ring Resonator (SRR) can be seen as a corresponding LC circuit, with its resonance properties determined by the equivalent inductance and capacitance. By controlling the capacitance of the SRR, many researches have demonstrated the tunability of LHM by different ways. LHM uses SRRs to create negative effective permeability over a particular frequency region and wire elements to produce negative effective permittivity in an intersecting frequency region. Therefore, despite the coupling effect this must be taken into account [4].

In other hand, particular dual-band and multi-band metamaterials have also been presented for multi frequency applications. It has also shown a dual-band and a multi-band metamaterial at microwave frequencies consisting of hexagonal SRR with asymmetric defects and dendritic structures, respectively [5]. Recently, some other researchers have also presented the dual-band metamaterials by using two magnetic resonators with different sizes or two different magnetic resonators.

All of the above mentioned metamaterials are based on immutable structures of the unit cell such as the SRR structures, resulting in a narrow band and not at all being tunable. A tunable multi-band metamaterial has been discussed by using micro-split SRR structures [7]. It just controlled the switching states of the micro-splits in the selected columns of micro-splits to achieve the tunable characteristic. Therefore, in order to design tunable dualband metamaterials, some other materials or structures should be used to achieve the tunable magnetic resonances or electronic resonances such as liquid crystals and superconductors [8]. Recently, some researchers proposed ferrite-based metamaterials, that is, substituting ferrites such as yttrium iron garnets (YIG) for the SRR The electromagnetic wave propagation properties of such metamaterials were numerically studied. The negative transmission and tunability characteristics was fabricated the metamaterial samples and explored experimentally [7, 9]. Here we present a novel design of implanting ferrites in the conventional metallic resonance metamaterials to 
achieve the tunable and dual-band characteristics. In this paper, we present basic types of tunable dual-band metamaterial composed of ferrite slabs and resonators, including SRR, the main goal of this work is the conception of tunable metamaterial filter based on dual band of ferrite $[5,6]$. The ferrite slabs provide one magnetic resonance frequency band under an applied magnetics bias and the metallic resonator provide another magnetic resonance frequency band, respectively. The continuous wires within the metamaterials provide the negative permittivity frequency band covering the above two magnetic resonance bands.

\section{Concept of Tunable Circular Split Ring Resonator Metamaterial}

To design a tunable circular split ring resonator metamaterial, we have analysed the tunable dual-band split ring resonator composed of ferrite slabs and SRR-wires from the schematic of unit cell with a cell dimension: $r=1.6$ $\mathrm{mm}, \mathrm{d}=0.1 \mathrm{~mm}, \mathrm{w}=0.2 \mathrm{~mm}$. This structure is presented in Figure 1. The SRR and wire, with thickness of 0.035 $\mathrm{mm}$, are placed in a Rogers T/duriod $5880\left(\varepsilon_{\mathrm{r}}=4.4, \tan \delta\right.$ $=0.0009$ ) substrate of $0.6 \mathrm{~mm}$ and separated of $0.25 \mathrm{~mm}$. After that the two sides of substrate are deposited with two ferrite slabs of $0.4 \mathrm{~mm}$. The ferrite slab has been modeled with a relative dielectric permittivity close to 13.02, a saturation magnetization equal to $\mathrm{Ms}=5000$ Gauss and a ferromagnetic resonance (FMR) line width $\Delta \mathrm{H}=73 \mathrm{Oe}$. The ferrite slab is magnetized perpendicularly to the propagation direction.

In the following section, we analyze the single-band metamaterials of SRR-wires structure to present the potential negative bands. The simulation results shown in Figure 2(a) indicate that the single-band SRR-wires metamaterial has a negative band centered at $15.13 \mathrm{GHz}$ negative band centered at $17.53 \mathrm{GHz}$ and a $3 \mathrm{~dB}$ bandwidth of $2 \mathrm{GHz}$. The transmission peak is $-0.59 \mathrm{~dB}$ at 16.7 GHz.

For ferrite-wire structure, the wire size is the same as above and the surrounding areas of the substrate are ferrite media. A magnetic bias of $200 \mathrm{KA} / \mathrm{m}$ acts on the ferrite slabs along the $\mathrm{y}$-axis. The simulation results in Figures 3(a) and (b) show clearly that there is a single band centered at $15.93 \mathrm{GHz}$ with $3 \mathrm{~dB}$ bandwidth of 2.1 $\mathrm{GHz}$. the transmission peak is -1.9 at $16.46 \mathrm{Ghz}$.

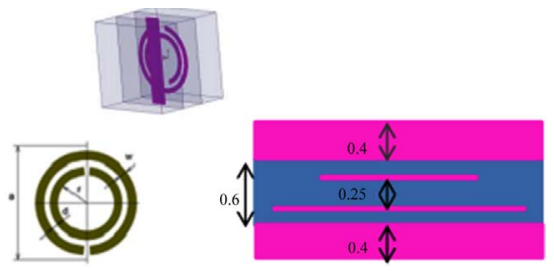

Figure 1. The schematic of dual-band metamaterial composed of ferrite slabs and SRR-wires structure.

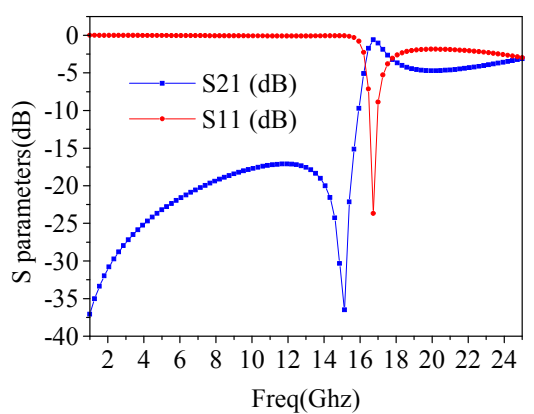

(a)

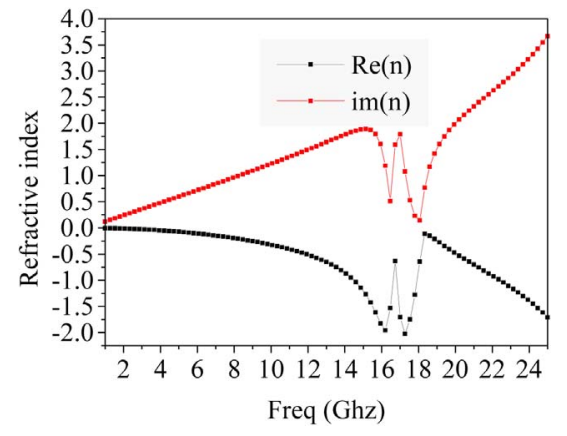

(b)

Figure 2. Result obtained for (a) Simulated transmission and reflection characteristics of single-band SRR-wires metamaterial (b) effective refractive index from the simulated S-parameters.

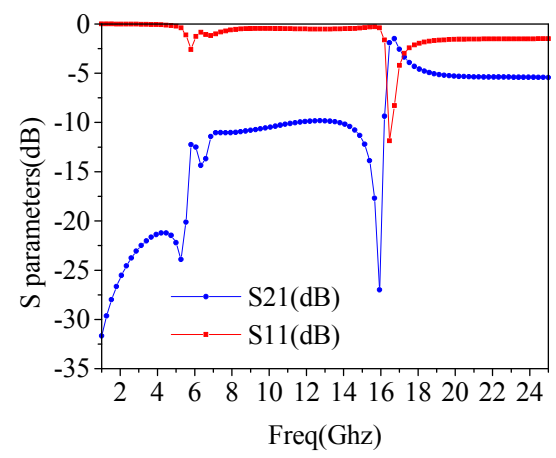

(a)

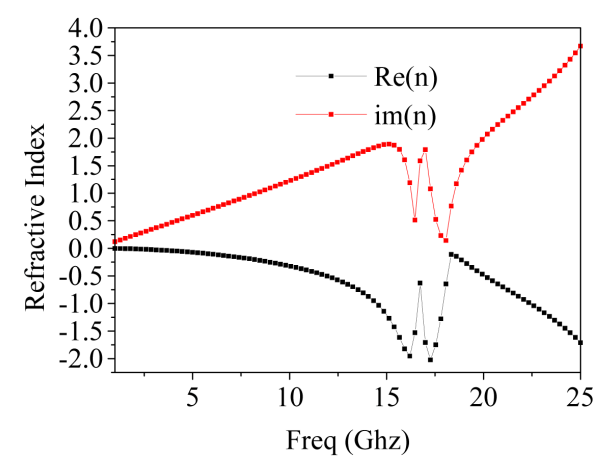

(b)

Figure 3. Single-band ferrites-wires metamaterial, simulated transmission and reflection (b) effective refractive index from the simulated S-parameters. 
Then the combined dual-band metamaterial as shown in Figure 1 is simulated. All the parameters of the unit cell are the same as above mentioned. The simulation results was obtained by the applied magnetics bias of 200 $\mathrm{kA} / \mathrm{m}$, are shown in Figure 4(a). It clearly shows that there are two bands centered at $5 \mathrm{GHz}$ and $16.3 \mathrm{GHz}$.

Comparing the dual-band metamaterial and the two single-band metamaterials, the first band contributed by ferrite slabs are not changed any more. The second band contributed by SRR in the combined dual-band metamaterial is higher than in the single-band SRR-wires metamaterial. This can be explained by the fact that the positive part of the effective permeability of ferrite slabs upper the negative part.

Lastly, to show the tunable characteristic of the dualband metamaterial, the unit cell is further simulated with different magnetic biases ranging from $150 \mathrm{kA} / \mathrm{m}$ to 300 $\mathrm{kA} / \mathrm{m}$, and the transmission characteristics are shown in Figure 5. It can be seen that the two pass bands are shifted. The first pass band is shifted from $5 \mathrm{GHz}$ to 8 $\mathrm{GHz}$ with the tuning rate of $1.875 \mathrm{MHz} / \mathrm{kA} / \mathrm{m}$ and the second pass band is shifted from $16 \mathrm{GHz}$ to $22 \mathrm{GHz}$ with the tuning rate of $2 \mathrm{GHz} / \mathrm{kA} / \mathrm{m}$.

The tuning level of the first pass band is larger than the second pass band. The transmission peaks and band

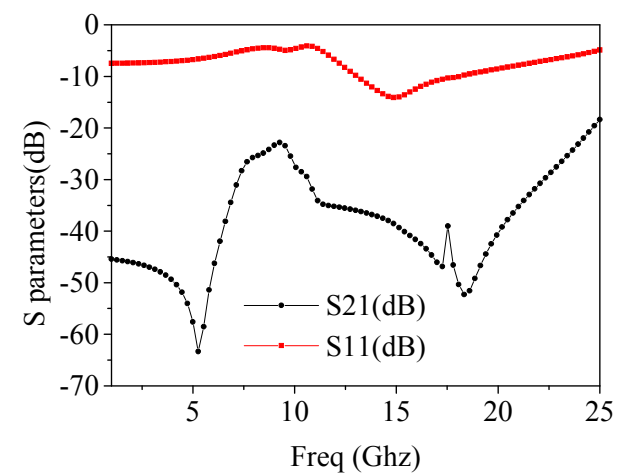

(a)

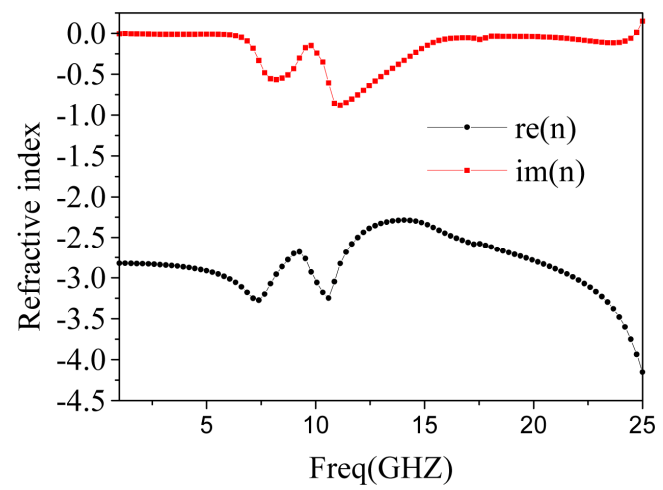

(b)

Figure 4. Result of the combination of dual-band ferritebased SRR-wires metamaterial (a) S-parameters; (b) refractive index.

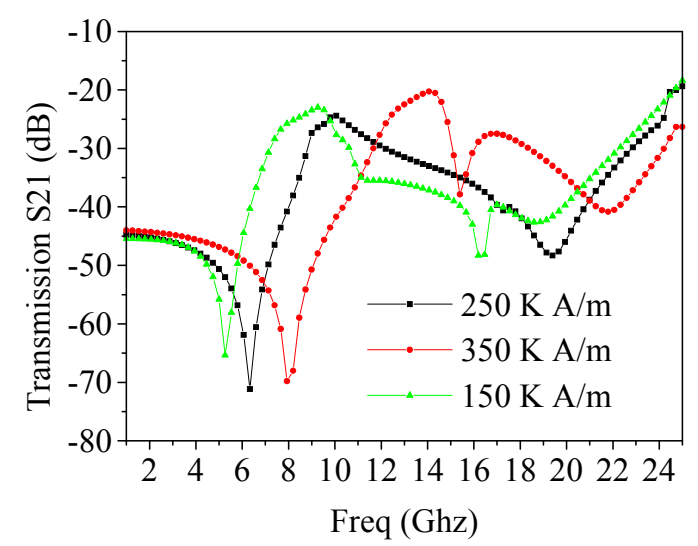

Figure 5. Numerical results of tenability of the ferrite-based SRR-wires metamaterial.

widths in the first pass band are not changed anymore and the second pass band is narrowed on increasing of the magnetic bias. This is because on increasing the magnetic bias, the negative permeability of ferrite slabs near the magnetic resonance shifted rapidly.

Figure 5 shows the tunable characteristic of the dualband metamaterial, the unit cell is further simulated with different magnetic biases ranging from $150 \mathrm{kA} / \mathrm{m}$ to 300 $\mathrm{kA} / \mathrm{m}$, and the transmission characteristics.

\section{Tunable Stop Band Filter Metamaterial Using Split Ring Resonator}

In this section, we discuss tunable filters using ferromagnetic materials in planar forms.

Firstly we analyze the proposed concept which can be used to implement higher order tunable stop band filters. Figure 6 shows a schematic of a typical microstrip stop band filter having six SRR resonators and a dual band of ferrite. The dimension of the SRR was the same as mentionedabove and the wire width is $\mathrm{w}_{\mathrm{w}}=2.16 \mathrm{~mm}$, gap length is $1 \mathrm{~g}=1.6 \mathrm{~mm}$ the total length of the filter is 1.5 $\mathrm{mm}$.

The filter is further simulated in different magnetic biases ranging from $150 \mathrm{kA} / \mathrm{m}$ to $300 \mathrm{kA} / \mathrm{m}$, and the transmission characteristics are illustrated in Figure 7 where it is shown that the two stop bands are shifted. The first stop-band is shifted from $3 \mathrm{GHz}$ to $5 \mathrm{GHz}$ with a tuning rate of $0.75 \mathrm{GHz} / \mathrm{kA} / \mathrm{m}$ and the second stop band is shifted from $5.75 \mathrm{GHz}$ to $7.8 \mathrm{GHz}$ with a tuning rate of $0.5 \mathrm{GHz} / \mathrm{kA} / \mathrm{m}$.

The tuning level of the first stop band is larger than the second stop band. The transmission peaks and band widths in the first stop band are not changed anymore and the second stop band is narrowed on increasing of the magnetic bias. This is because on increasing the magnetic bias, the negative permeability of ferrite slabs near the magnetic resonance shifted rapidly. 
The second type of stop band filter consists of three SRR and wire with ferrite slabs (Figure 8).

The filter is further simulated in different magnetic biases ranging from $150 \mathrm{kA} / \mathrm{m}$ to $300 \mathrm{kA} / \mathrm{m}$, and the transmission characteristics are illustrated in Figure 9. The stop band is shifted from $6.6 \mathrm{GHz}$ to $7.6 \mathrm{GHz}$ with a tuning rate of $0.875 \mathrm{MHz} / \mathrm{kA} / \mathrm{m}$.

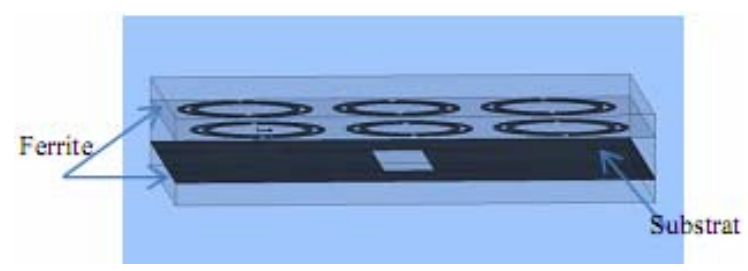

(a)

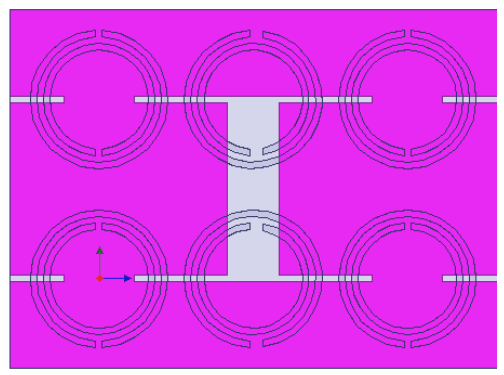

(b)

Figure 6. (a) Tunable filter configuration with the ferrite slabs; (b) Its cross section.

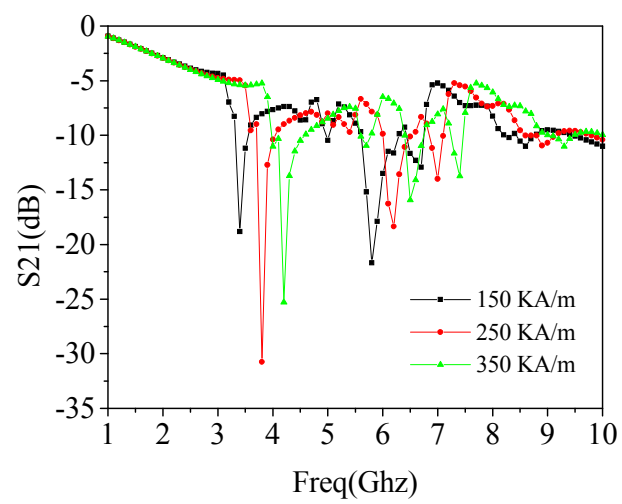

Figure 7. Simulated S21 results of filter using ferrite at $\mathbf{H}_{0}=$ $100 \mathrm{kA} / \mathrm{m}, H_{0}=150 \mathrm{kA} / \mathrm{m}$ and $H_{0}=350 \mathrm{kA} / \mathrm{m}$.

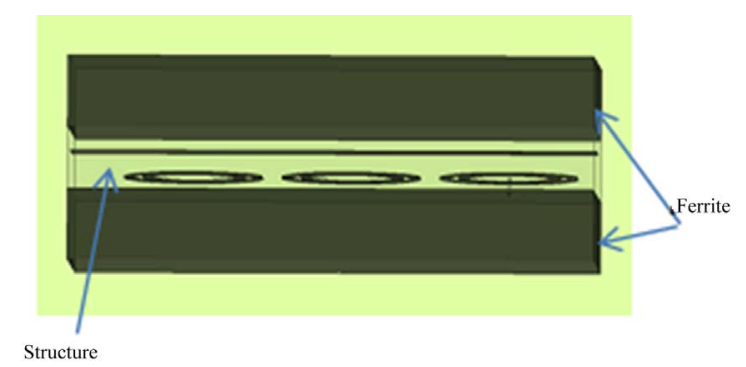

Figure 8. Structure of a tunable stop band filterwith three SRR and wire and dual band of ferrite.

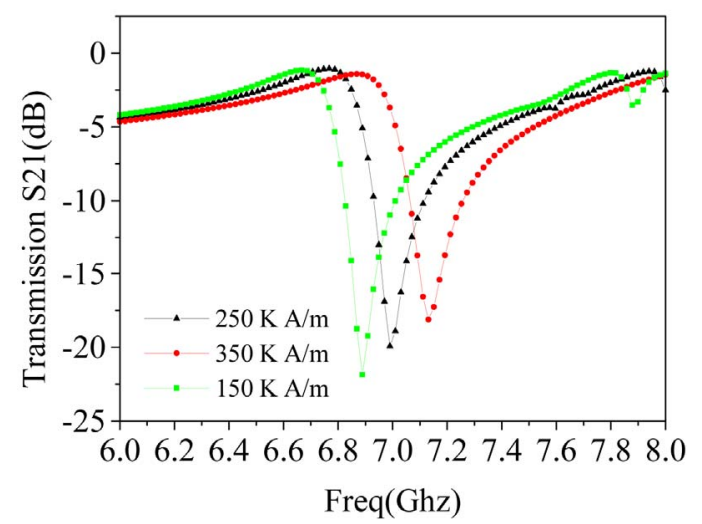

Figure 9. Simulated S21 results of filter using ferrite at $\mathbf{H}_{0}=$ $100 \mathrm{kA} / \mathrm{m}, H_{0}=150 \mathrm{kA} / \mathrm{m}$ and $H_{0}=350 \mathrm{kA} / \mathrm{m}$.

\section{Conclusion}

In this paper, we have designed a tunable dual-band metamaterial composed of ferrites providing negative permeability band, and magnetic resonators, including SRR and continuous wire providing the negative permitivity. A novel frequency-tunable stop band filter based on SRR-wire elements was proposed and the corresponding simulation results were presented. It was demonstrated that the band-pass response could be dynamically tuned to different frequency bands using ferrite.

\section{REFERENCES}

[1] V. G. Veselago, "The Electrodynamics of Substances with Simultaneously Negative Values of $\varepsilon$ and $\mu$," Soviet Physics Uspekhi, Vol. 10, No. 4, 1968, pp. 509-514. doi:10.1070/PU1968v010n04ABEH003699

[2] J. B. Pendry, "Negative Refraction Makes a Perfect Lens," Physical Review Letters, Vol. 85, No. 18, 2000, pp. 39663969. doi:10.1103/PhysRevLett.85.3966

[3] D. R. Smith, W. J. Padilla, D. C. Vier, S. C. Nemat-Nasser and S. Schultz, "Composite Medium with Simultaneously Negative Permeability and Permittivity," Physical Review Letters, Vol. 84, No. 18, 2000, pp. 4184-4187. doi:10.1103/PhysRevLett.84.4184

[4] Y. Huang, G. Wen, T. Li and K. Xie, "Low-Loss, Broadband and Tunable Negative Refractive Index Metamaterial," Journal of Electromagnetic Analysis \& Applications, Vol. 2, 2010, pp. 104-110. doi:10.4236/jemaa.2010.22015

[5] L. Kang, Q. Zhao, H. J. Zhao and J. Zhou, "Ferrite-Based Magnetically Tunable Left-Handed Metamaterial Composed of SRRs and Wires," Optics Express, Vol. 16, No. 22, 2008, pp. 17269-17275. doi:10.1364/OE.16.017269

[6] T. H. Feng, Y. H. Li, H. T. Jiang, W. X. Li, F. Yang, X. P. Dong and H. Chen, "Tunable Single-Negative Metamaterials Based on Microstrip Transmission Line with Varactor Diodes Loading," Progress in Electromagnetics Research, Vol. 120, 2011, pp. 35-50.

[7] H. J. Zhao, J. Zhou, Q. Zhao, B. Li, L. Kang and Y. Bai, "Magnetotunable Left-Handed Material Consisting of Yt- 
trium Iron Garnet Slab and Metallic Wires," Applied Physics Letters, Vol. 91, 2007, Article ID: 131107.

[8] Q. Zhao, L. Kang, B. Du, B. Li, J. Zhou, H. Tang, X. Liang and B. Zhang, "Electrically Tunable Negative Permeability Metamaterials Based on Nematic Liquid Crystals," Applied Physics Letters, Vol. 90, 2007, Article ID:
011112 .

[9] Y. X. He, P. He, S. D. Yoon, P. V. Parimi, F. J. Rachford, V. G. Harris and C. Vittoria, "Tunable Negative Index Metamaterial Using Yttrium Iron Garnet," Journal of Magnetism and Magnetic Materials, Vol. 313, No. 1, 2007, pp. 187-191. doi:10.1016/j.jmmm.2006.12.031 